\title{
Predicting Harmonic Distortion of Multiple Converters in a Power System
}

\author{
P. M. Ivry, O. A. Oke, D. W. P. Thomas, and M. Sumner \\ Department of Electrical and Electronics Engineering, Electrical Systems and Optics Research Group, \\ University of Nottingham, Nottingham, UK \\ Correspondence should be addressed to P. M. Ivry; preyeivry@mail.ndu.edu.ng
}

Received 5 March 2017; Accepted 24 April 2017; Published 5 June 2017

Academic Editor: Raj Senani

Copyright (C) 2017 P. M. Ivry et al. This is an open access article distributed under the Creative Commons Attribution License, which permits unrestricted use, distribution, and reproduction in any medium, provided the original work is properly cited.

\begin{abstract}
Various uncertainties arise in the operation and management of power systems containing Renewable Energy Sources (RES) that affect the systems power quality. These uncertainties may arise due to system parameter changes or design parameter choice. In this work, the impact of uncertainties on the prediction of harmonics in a power system containing multiple Voltage Source Converters (VSCs) is investigated. The study focuses on the prediction of harmonic distortion level in multiple VSCs when some system or design parameters are only known within certain constraints. The Univariate Dimension Reduction (UDR) method was utilized in this study as an efficient predictive tool for the level of harmonic distortion of the VSCs measured at the Point of Common Coupling (PCC) to the grid. Two case studies were considered and the UDR technique was also experimentally validated. The obtained results were compared with that of the Monte Carlo Simulation (MCS) results.
\end{abstract}

\section{Introduction}

Controllable small distribution networks containing closed assemblage of distributed generators, Renewable Energy Systems (RES), storage systems, and loads are becoming increasingly common in Electrical Power Systems (EPS). The optimization of RES, such as wind turbines and photovoltaics, has been successful thus far. This can be attributed to the advancement and use of power electronic converters, which help to achieve higher power and voltage level operation in wind turbines and photovoltaics.

In recent years, the Voltage Source Converter (VSC) is popularly used and has enjoyed more attention than other converters due to its better controllability and fast switching responses $[1,2]$. Nevertheless, VSCs generate harmonic voltages and currents that are transmitted to the rest of the grid. These harmonics may cause malfunction or damage of the power system and equipment on the system.

On an EPS, harmonics can significantly increase and become difficult to predict where variations are present in certain factors like the operating conditions (output power) or system parameters (grid voltage background distortion) [3]. Furthermore, in a case where many VSCs are connected to the EPS, the net effect of the harmonics on the EPS's Power Quality (PQ) becomes more challenging to predict as harmonics do not add up arithmetically. This and the inherent stochastic nature of harmonics necessitate the use of statistical techniques in predicting the cumulative harmonic distortion level of power converters in an EPS. The use of statistical techniques was also suggested by the IEEE Probabilistic Aspects Task Force on Harmonics for quantifying harmonic distortions [4].

Statistical techniques, such as the Monte Carlo Simulation (MCS) [5], have been extensively used as a common approach in predicting the harmonic distortion level of power converters [6,7]. However, it requires tens of thousands of simulations to obtain an accurate prediction and this affects the feasibility of using such approach for systems containing large number of VSCs.

In some previous studies [8-14], an analytical approach was used to predict the level of harmonics generated by power converters. The studies represented harmonic vectors as phasors having random amplitudes and angles. The probability density functions (pdfs) of the phasors were obtained and represented in the rectangular coordinates for the convenience of adding phasors. In cases of a large number 
of harmonic sources/loads the harmonic phasor's pdfs are then vectorially summed. The studies predicted harmonics in terms of low harmonic orders such as the 3rd, 5th, 7th, and 11th harmonic orders. This may be due to the type of power converter utilized (6/12 pulse converters) and their associated harmonics generated. In a VSC system, the majority of the harmonics appear at the switching frequency and multiplies of the switching frequency $[1,2]$. Another way to quantify the harmonics would be to use the Total Harmonic Distortion (THD) [2].

Furthermore, an analytical approach usually entails assumptions to handle the complex interaction of a large number of random harmonic quantities $[15,16]$ and practical converter systems were usually simplified and represented by mathematical formulas to accommodate the approach as seen in [8-14]. However, other methods which do not require these simplifications or assumptions in designing the systems/converters or in generating random occurrences can be deployed. They include the Monte Carlo Simulation (MCS) $[5,17,18]$, Unscented Transform (UT) [19, 20], Point Estimate Method (PEM) [21], and Univariate Dimension Reduction (UDR) [22, 23]. For instance, in Probabilistic Load Flow (PLF) studies, some of these methods have been utilized. The UDR which has proved successful for problems with complex and large number of statistical variation will be utilized in the probabilistic harmonic analysis because of its ability to drastically reduce computational cost, time, and burden. In this study, practical VSC models are simulated with a full switching model.

In VSC design and utilization, the interfacing inductor value depends on the VSC switching frequency to achieve adequate attenuation of harmonics and it is usually chosen with a tradeoff between harmonic attenuation capability and filter cost. Furthermore, various formulas [24-28] can be used in estimating the inductance value with each giving a different filter size, thus contributing to the uncertainty. In most RES, output power is variable. For wind turbine systems, the output power is a function of the wind speed, while, for photovoltaics, output power depends mainly on incident sun rays on the photovoltaic cells. This gives rise to further uncertainty as wind speed/sun light varies with time, day, season, and place. All these uncertainties have to be properly accounted for to ensure harmonic distortion within the EPS is within prescribed limits.

This paper presents a method for predicting current harmonics at the PCC of an EPS in the presence of uncertainties in the filter parameter and operating power of multiple VSCs. The level of harmonic distortion is quantified using statistical evaluators such as the mean and standard deviation. The Univariate Dimension Reduction (UDR) for 3 and 5 points' approximation is utilized. It is utilized as an alternative to the Monte Carlo Simulation (MCS) in predicting the harmonics distortion level of multiple connected VSCs because of its significant reduction of computational cost, time, and burden associated with the MCS. The results obtained and the accuracy of the UDR for each of the cases were compared with the MCS and presented in the sections below. The results for the UDR technique were also validated using a laboratory experiment.

\section{Unscented Transform and Univariate Dimension Reduction}

2.1. Unscented Transform (UT). The UT works by approximating a nonlinear mapping by a set of selected points called sigma points. The sigma points are developed using the moments of the distribution functions pdf, and the weighted average of the sigma points produces the expectation of the mapping [29]. The UT has been utilized in nonlinear problems in electromagnetic compatibility $[19,20,30]$ and medical statistics amongst other fields [22]. This method can be used in approximating a continuous distribution function with pdf $w(x)$ as a discrete distribution using deterministically chosen points called sigma points $\left(S_{i}\right)$ and weights $\left(w_{i}\right)$ such that the moments of both distributions are equal [31]. This is mathematically represented in

$$
E\left(x^{k}\right)=\int x^{k} w(x) d x=\sum_{i} w_{i} S_{i}^{k}
$$

$S_{i}$ contains the location of the abscissas at which the function $f(x)$ is to be evaluated while $w_{i}$ are the weighting coefficients which when multiplied by $S_{i}$ give an approximation to the integral of $f(x) . k$ represents the moments of the expectation $(E)$ where $k=1$ implies the mean value and $k=2$ implies variance.

The Gaussian quadrature technique is applied to solve (1) such that the integration points for integrating $f(x)$ correspond to the desired sigma points $S_{i}$. Hence, for a function $f(x)$ assumed as a polynomial, with pdf (weighting function) $w(x)$, the nonlinear mapping for the expectation is given as

$$
E[f(x)]=\int_{-\infty}^{\infty} f(x) w(x) d x=\sum_{i} w_{i} f\left(S_{i}\right) .
$$

The sigma points of the distribution function can be obtained as the roots of its associated orthogonal polynomial when (1) or (2) is integrated using Gaussian quadrature. This method can be easily applied, as most common distributions have known classical orthogonal polynomials associated with them. The associated orthogonal polynomials for some distribution functions can be found in [32].

2.2. Univariate Dimension Reduction (UDR). The procedure discussed in the section above is only directly applicable when obtaining individual sigma points and weights. For problems involving more than one variable $(N)$, the simplest technique is based on tensor product of the individual sigma points $(n)$, giving the number of evaluations $E_{v}$ as

$$
E_{v}=n^{N}
$$

Unfortunately, the technique is plagued by the curse of dimensionality problem as the number of variables increases. For example, using 5 sigma points in (3), 5 variables will require 3,125 evaluations while, for 10 variables, $E_{v}=9,765,625$. The dimension reduction technique is thus employed in this work. 
The dimension reduction $[22,29,33,34]$ is an approximate technique for estimating the statistical moments of an output function. The technique involves an additive decomposition of an $N$-dimensional function involving $n$ dimensional integral into a series sum of $D$-dimensional functions such that $D<N$. It provides a means of efficiently combining the sigma points and weights for a large number of variables such that the number of evaluation points can be minimized $[22,29]$. For $D=1$ the method is referred to as the Univariate Dimension Reduction (UDR) while it is called bivariate dimension reduction for $D=2$.

The UDR method has been utilized in stochastic mechanics [23] and probabilistic load flow studies [22, 35, 36]; however, it has been less applied to harmonic analysis. The UDR method is briefly described below while a detailed mathematical derivation of the techniques can be found in [33].

With the UDR, the main function, $f(\mathbf{x})$, is decomposed into a summation of one-dimensional functions such that

$$
\begin{aligned}
f(\mathbf{x}) \cong & \sum_{i=1}^{N} f\left(\bar{x}_{1}, \ldots, \bar{x}_{i-1}, x_{i}, \bar{x}_{i+1}, \ldots, \bar{x}_{N}\right) \\
& -(N-1) f\left(\bar{x}_{1}, \ldots, \bar{x}_{N}\right),
\end{aligned}
$$

where $\mu_{i}$ is the mean of the $i$ th random variable.

The resultant function in (4) can easily be integrated as only one randomly distributed variable is present at every instance while the others are held constant at their mean values. The moments of the function are approximately the same as those of the decomposed function as represented in

$$
\begin{aligned}
E[f(\mathbf{x})] \cong & E[\widehat{f}(\mathbf{x})] \\
\cong & \sum_{i=1}^{N} E\left[f\left(\bar{x}_{1}, \bar{x}_{2}, \ldots, x_{i}, \bar{x}_{N}\right)\right] \\
& -(N-1) E\left[f\left(\bar{x}_{1}, \ldots, \bar{x}_{N}\right)\right] .
\end{aligned}
$$

The same procedure is applied for obtaining the higher order moments.

The number of evaluations $\left(E_{\gamma}\right)$ required for an $N$ dimensional function using $n$ estimation (sigma points) for the UDR is given in [33]

$$
E_{v}=(n \times N)+1 .
$$

For problems where all random variables are symmetrical and $n$ (sigma points) is odd, the estimation points for the UDR can be further reduced to (7) while still maintaining the same level of accuracy. For clarity, this will be referred to as reduced UDR (rUDR).

$$
E_{v}=((n-1) \times N)+1 .
$$

This is clearly a substantial saving in computational time over the alternative of $n^{N}$ evaluations.

\section{Uncertainty Representation}

The appropriate design and sizing of the system filter have a great impact on the amount of distortion seen at the grid side of the converter. If not designed properly, it can affect the systems stability. When an RES is integrated to the grid, one of the main concerns is to reduce the harmonic current injected into the grid [24]. In designing harmonic filters for converters, the maximum ripple current should be less than $20 \%$ of the rated current [24]. This current is a function of the inductance $L_{f 1}$, the switching frequency, and the DC link voltage. However, to achieve a decrease of the ripple current, the inductance is the most flexible parameter since increasing the switching frequency affects the system efficiency. The value of the inductance can be obtained using

$$
L_{f 1}=\frac{1}{8} \times \frac{V_{\mathrm{dc}}}{f_{\mathrm{sw}} \cdot \Delta \widehat{I}_{L \max }},
$$

where $f_{\text {sw }}$ is the switching frequency, $V_{\mathrm{dc}}$ is the dc voltage, and $\Delta \widehat{I}_{L \max }$ is the peak ripple of the maximum rated load current.

In deciding the filter inductance value, another solution is to choose the value relative to the total system inductance. It was suggested in $[25,28]$ that the total system inductance should be about $10 \%$ of the base inductance value. Since no fixed value can be used but that which is determined at the discretion of the designer, the interfacing inductor $L_{f}$ can be viewed as a stochastic variable such that the optimal size cannot be clearly determined. The value of the inductance will be in a range, howbeit as a percentage of $\Delta \widehat{I}_{L \max }$. The value of the inductance can thus be represented using the uniform distribution such that an optimal value can be easily predicted. Hence, (9) can be substituted into (8) to account for this variation.

$$
\Delta \widehat{I}_{L \max }=(5-20 \%) \times I_{\text {rated }}
$$

and $L_{f 2}$ is calculated as

$$
L_{f 2}=\alpha L_{f 1},
$$

where $\alpha$ is a factor limiting $L_{f 2}$ to be less than $L_{f 1}$.

The VSC harmonic filter is designed using (8)-(14). The value for the filter capacitor $\left(C_{f}\right)$ can be obtained using

$$
C_{f}=15 \% \times \frac{P_{\text {rated }}}{3 \cdot 2 \pi f_{m} \cdot V_{\text {rated }}^{2}},
$$

where $C_{f}$ is calculated by limiting the total reactive power required by the capacitor to be within $15 \%$ of the total rated active power.

It is important to consider resonant frequency of an LC or LCL filter as they usually require a damping element to maintain stability and to ensure optimum operation [24].

The resonant frequency is usually within $10 f_{n}<f_{\text {res }}<$ $f_{\text {sw }} / 2$.

For an LC filter, the resonant frequency is calculated from

$$
\omega_{\text {res }}=\frac{1}{\sqrt{L_{f 1} C_{f}}} .
$$

For an LCL filter, it is

$$
\omega_{\text {res }}=\sqrt{\frac{L_{f 1}+L_{f 2}}{L_{f 1} L_{f 2} C_{f}}} .
$$



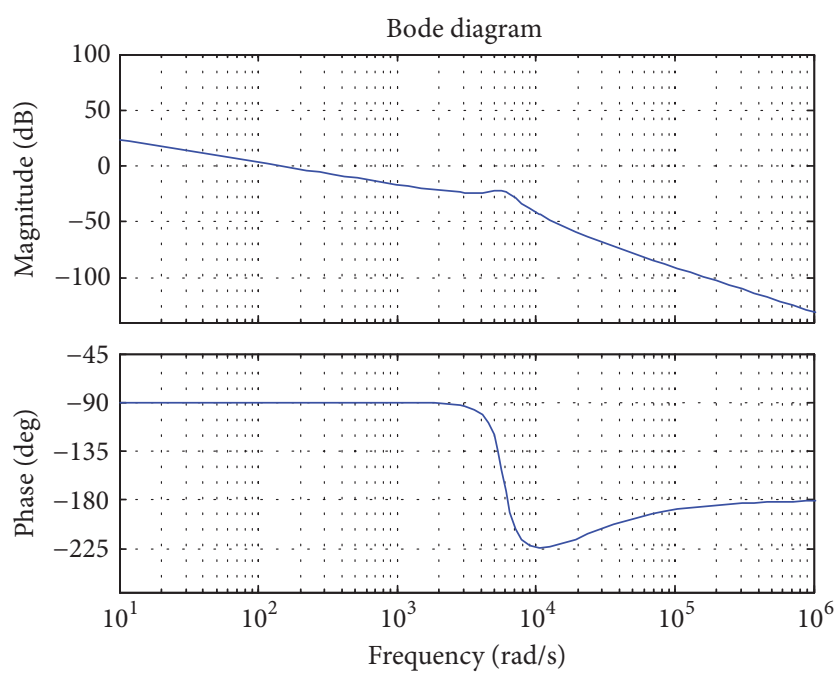

Figure 1: Bode plot of designed filter.

The damping resistor is calculated using [24]

$$
R_{d}=\frac{1}{3 \cdot \omega_{\mathrm{res}} \cdot C_{f}}
$$

Figure 1 shows the bode plot of the designed filter and highlights the effectiveness of the filter.

Similarly, other uncertainties that emanate from various RES due to the dependence of their power output on several random factors such as the weather conditions have to be represented. Several models have been proposed in literatures for modeling these uncertainties; wind speed has been modeled using the Weibull distribution, Rayleigh distribution, normal distribution, and so forth [37]. For simplicity, the Power Variation is modeled using the uniform distribution.

\section{EPS Structure}

The studied RES system comprises the DC Power Source, DC capacitor, RES interfacing VSC, VSC reactors/filter, grid impedance, and 3-phase grid voltage (representing further parts of the grid). Figure 2 shows multiple numbers of VSCs connected in parallel and interfaced to the grid at the PCC via the grid impedance. The system was simulated using PLECS ${ }^{\text {тм }}$ [38] and MATLAB Simulink ${ }^{\mathrm{TM}}$ [39] simulation tools.

The VSC is modeled as a 2-level VSC and the switching pattern is based on the Sinusoidal Pulse Width Modulation (SPWM) as in [2]. The VSC is controlled based on the commonly used instantaneous power theory [1] using PI controllers. The first PI controller controls the DC capacitor voltage to remain as regulated. The output of the regulated voltage gives the reference $I_{d \text { ref }}$ that is required to regulate the active current component which in turn controls the active power $[1,3]$. The second current PI controller controls the reactive current component $I_{q \text { ref }}$. For this study $I_{q \text { ref }}=I_{q}=0$ [1]. The control scheme used in [3] was adopted for each of the VSCs.

\section{UDR Harmonic Prediction Process}

A summary of the main steps for implementing the UDR technique for predicting current and voltage harmonic distortion at the PCC of the EPS is highlighted below.

Step 1. Identify all randomly varying functions within the system (e.g., power, filter inductance) and obtain their probability distribution functions.

Step 2. Compute the sigma points and weights using Univariate Dimension Reduction technique.

Step 3. Input the EPS data including the sigma points and weights obtained in Step 2.

Step 4. Run the EPS simulation while obtaining necessary statistical data for the output variables (current and voltage THD).

Step 5. Compute the statistical data for the output variables using the measured output data values and weights of the UDR (e.g., mean current THD).

Step 6. Display statistical data of output variables (e.g., mean current and voltage THD).

\section{Simulation Results}

Two (2) studies are considered in predicting the level of harmonic distortion based on the variation of output power and filter inductor value. In the first study, the effect of filter inductor value $L_{f 1}$ on the level of harmonic distortion in the current at the PCC using multiple VSCs is examined. For this system, $L_{f 1}$ was calculated using techniques in $[24,40]$ which gives a value of $5.76 \mathrm{mH}$ which is typical for systems of this level. Based on this and assuming the filter inductor value can be modeled using the uniform distribution, the value was chosen to be between $3.46 \mathrm{mH}$ and $8.06 \mathrm{mH}$. This range was chosen assuming a $\pm 40 \%$ tradeoff between harmonic filter effectiveness and cost of filter inductor.

In the second study, the effect of output Power Variations on the level of harmonic distortion is considered. The output power is assumed to vary randomly within a range following the uniform distribution between $25 \%$ and $100 \%$ of its rated power. The point here is to show wide variability of the RES output power.

In the considered cases, 1000 simulations were carried out for the MCS to ensure accuracy. This is sufficient as this ensures a 95\% confidence interval that the errors in the mean THD values are less than $3 \%$ as calculated using (15). The mean and standard deviation values utilized in this calculation were obtained after running the simulations and then used to check against the $95 \%$ CI.

$$
\begin{aligned}
95 \%(\mathrm{CI}) & =\bar{x} \pm Z \frac{\sigma}{\sqrt{n}} \\
\mathrm{SE} & =\frac{\sigma}{\sqrt{n}},
\end{aligned}
$$




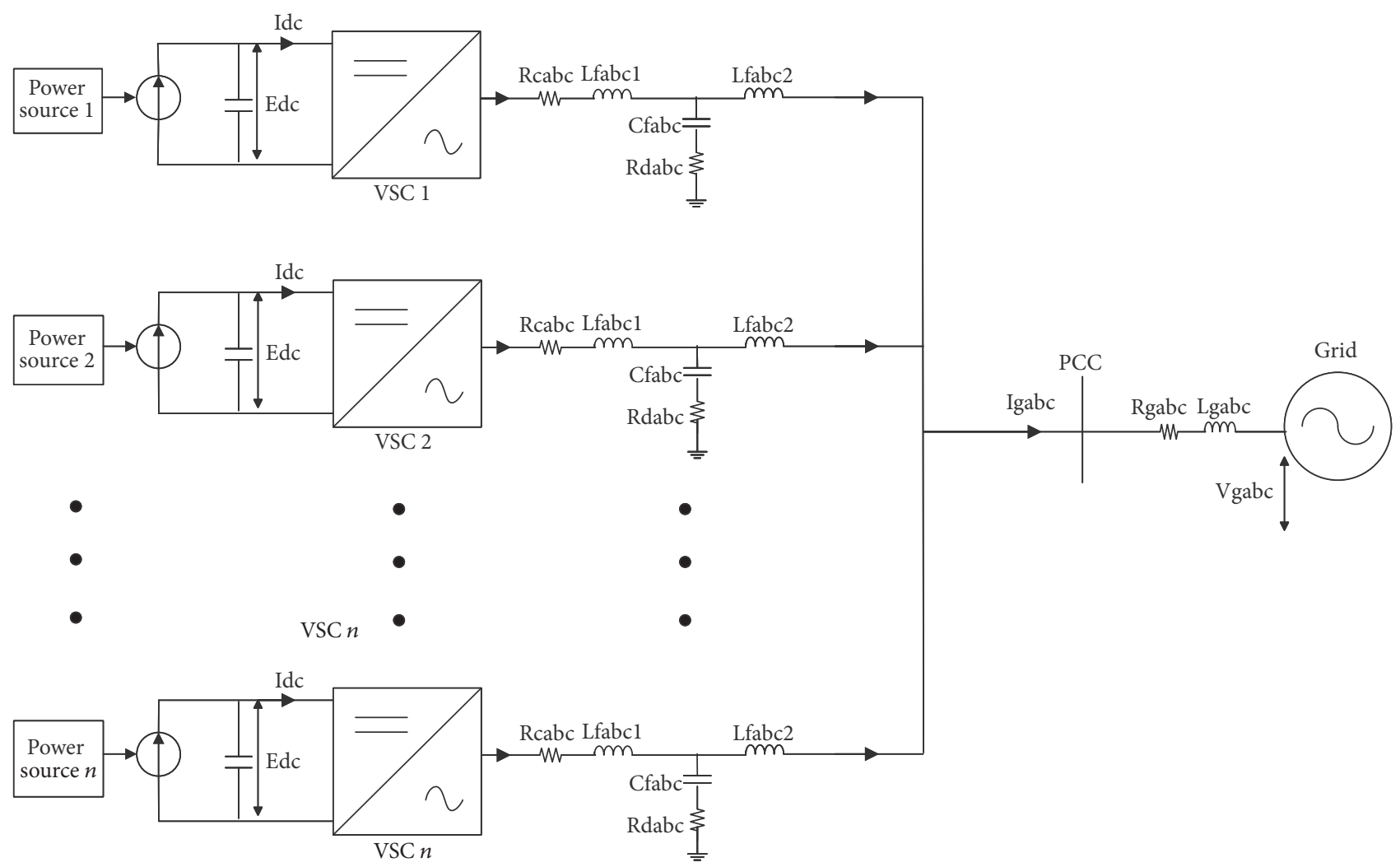

Figure 2: Multiple VSCs connected to a common point to the grid.

where CI implies the confidence interval, SE is the standard error of the mean, $n$ is the number of samples, $\bar{x}$ implies the mean value of the samples, $\sigma$ is standard deviation of the samples, and $Z=1.96$ the constant representing 95\% CI, where the SE for 2 VSCs at 1000 simulations gives 0.04 using the values for 2 VSCs in Figure 3.

To show the adequacy of the chosen number of simulations used, Figure 3 presents the results for the mean current THD for a system with 2 VSCs using 10, 100, 1000, 5000, and 10000. The simulations were carried out using a Windows 7, 32-bit operating system, i3 processor 4 GB RAM PC.

The 3 and 5 points' approximated rUDR were utilized in predicting the THD of the power converters in the EPS. The obtained results were then compared with that of the MCS. The results obtained in terms of the mean and standard deviation of the THD and the accuracy of the methods for each of the cases are discussed in the sections below.

6.1. Effect of Filter Variation on Current THD. The mean current and voltage THD arising from $L_{f}$ variations using 1 to 10 VSCs are presented in Figures 5 and 6, respectively. Figure 4 shows the amount of time saved when the rUDR methods were utilized and compared with the MCS. For 10 VSCs the amounts of simulation time for the rUDR 3 points, rUDR 5 points, and MCS were approximately $1100 \mathrm{~s}$, $2100 \mathrm{~s}$, and $52000 \mathrm{~s}$, respectively.

$$
\text { Computational Time Saved }(\%)=\frac{t_{\mathrm{MCS}}-t_{\mathrm{rUDR}}}{t_{\mathrm{MCS}}} \text {. }
$$

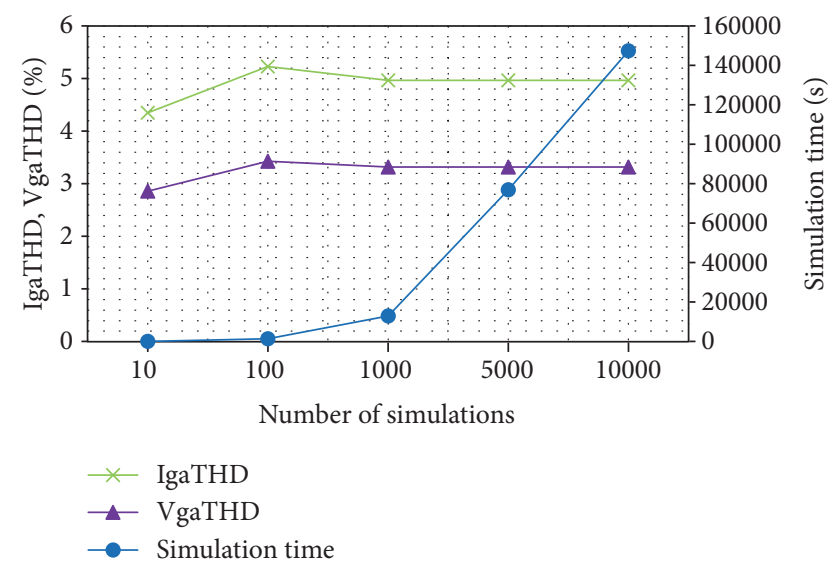

FIGURE 3: THD result for 2 VSCs connected in parallel.

From Figure 5, it can be observed that the impact of the variation of $L_{f}$ on the current THD progressively reduces as the number of VSC increases. This is as expected, since the total number of filters in the system increases as the number of VSC increases, thus limiting the current distortion. Also, with increased number of VSCs, there is a higher probability of harmonic cancellation due to current diversity and attenuation factor [24, 41, 42].

It is observed that the rUDR methods closely predict the current and voltage THD as the curve follows the same graph trend as that of the MCS. With 10 VSCs, there seems to be 


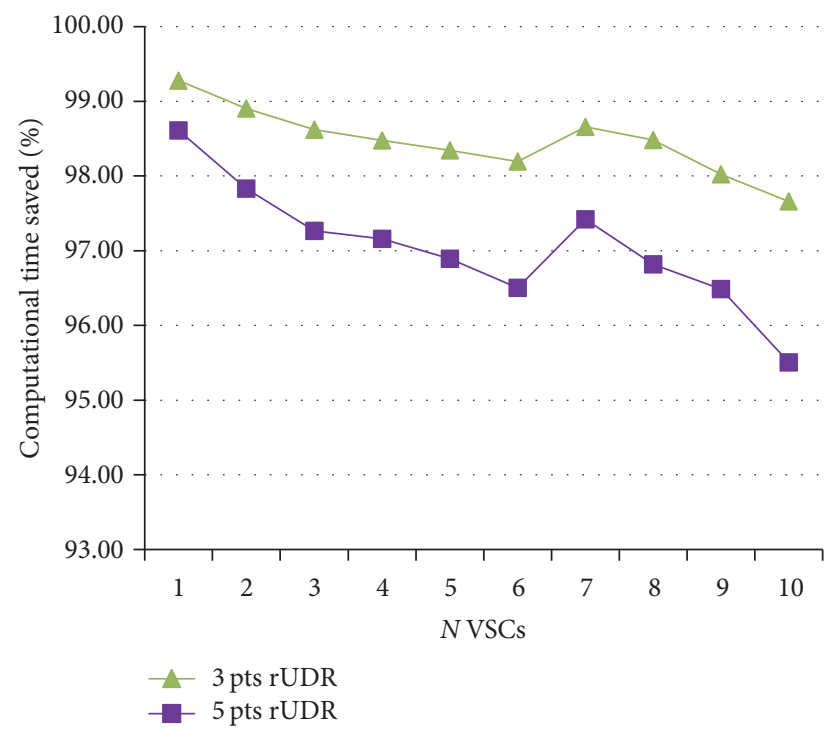

FIgURE 4: Computation time saved by rUDR 3 pts and rUDR 5 pts.

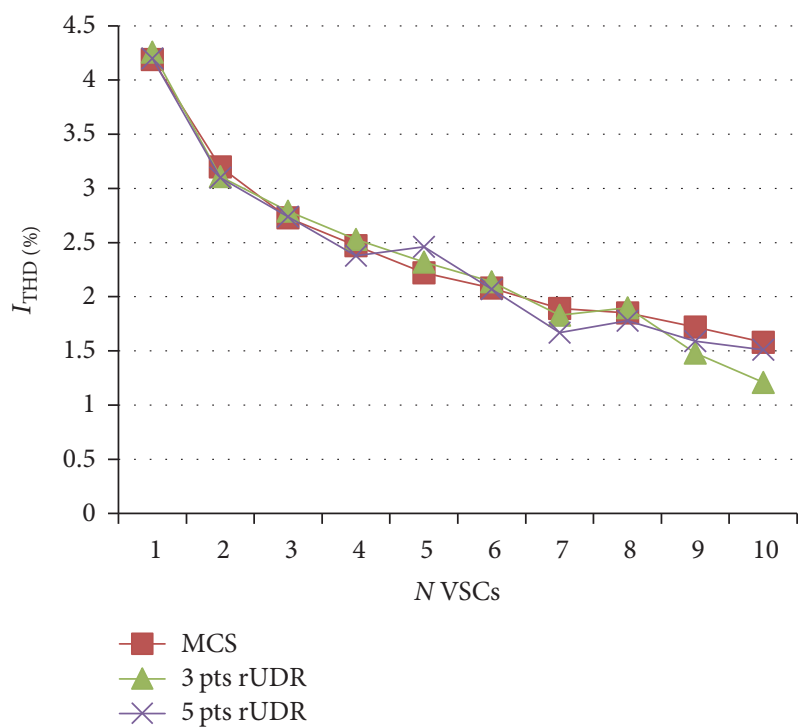

Figure 5: Predicted IgaTHD using MCS, rUDR 3 pts, and rUDR 5 pts under Filter Variation.

a slight underestimation with the UDR methods which could be accepted considering the amount of computation time saved in obtaining the results (Figures 5 and 6).

6.2. Effect of Power Variation on Current THD. The impact of operating Power Variation on the current and voltage THD seen at the PCC of the EPS was also investigated. The predicted current and voltage THD using the UDR and MCS approach are presented in Figures 7 and 8. The impact of Power Variation on the current THD is more significant than the case of $L_{f 1}$ variation as evident in Figure 7 . However, as with the previous case, the current THD is observed to reduce with the increase of the number of VSCs. The rUDR methods

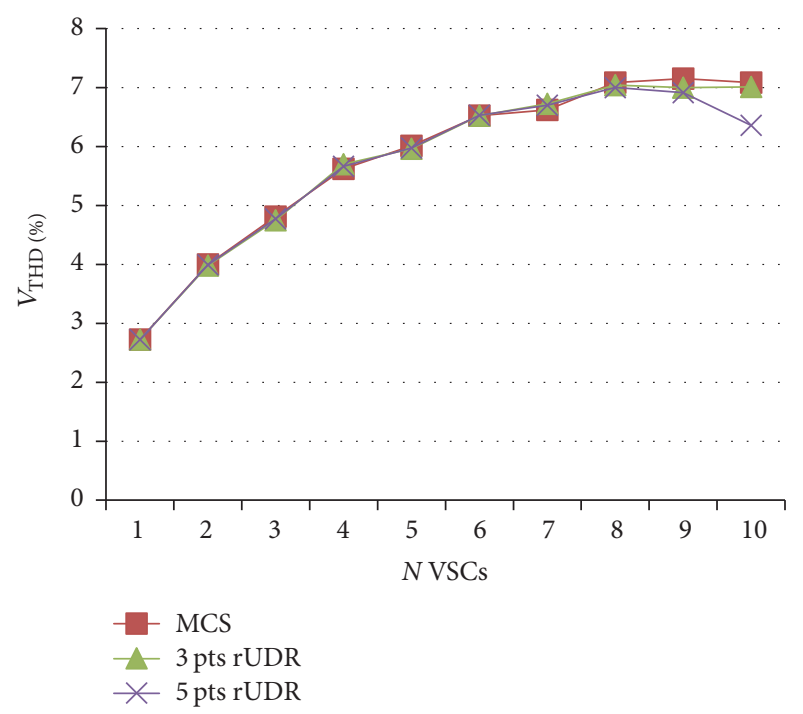

FIgUre 6: Predicted VgaTHD using MCS, rUDR 3 pts, and rUDR 5 pts under Filter Variation.

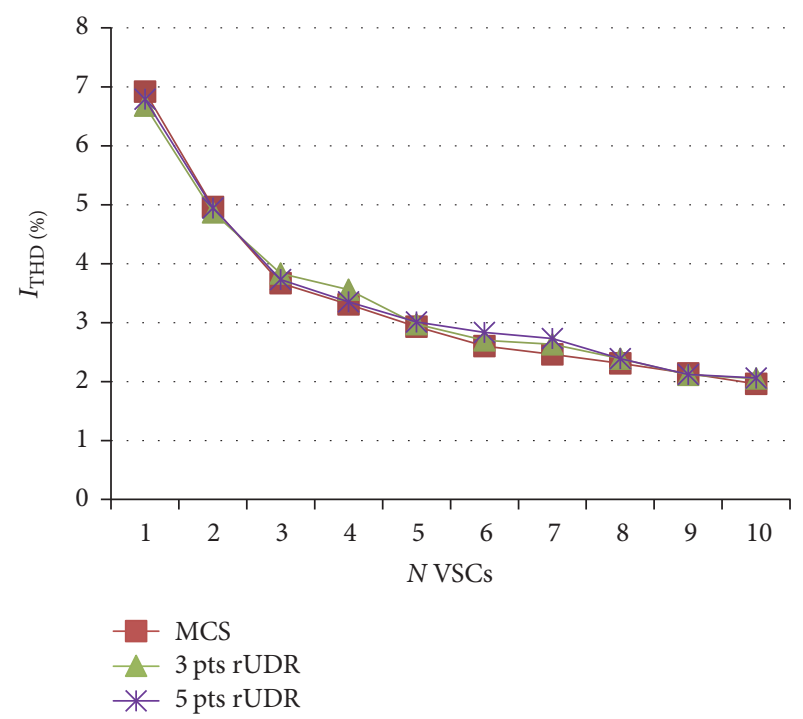

FIgure 7: Predicted IgaTHD using MCS, rUDR 3 pts, and rUDR 5 pts under Power Variation.

produced accurate results and the graph trends are similar to the MCS.

\section{Experimental Validation of the UDR Technique}

The aim of this experiment is to validate the efficiency of the UDR technique in predicting the harmonic distortion of VSCs in the presence of uncertainty for a small Electrical Power System (EPS). The parameters of which are given in Table 1.

Figure 9 shows the schematic of the laboratory setup. The experiment was conducted using 3 VSCs, a programmable 


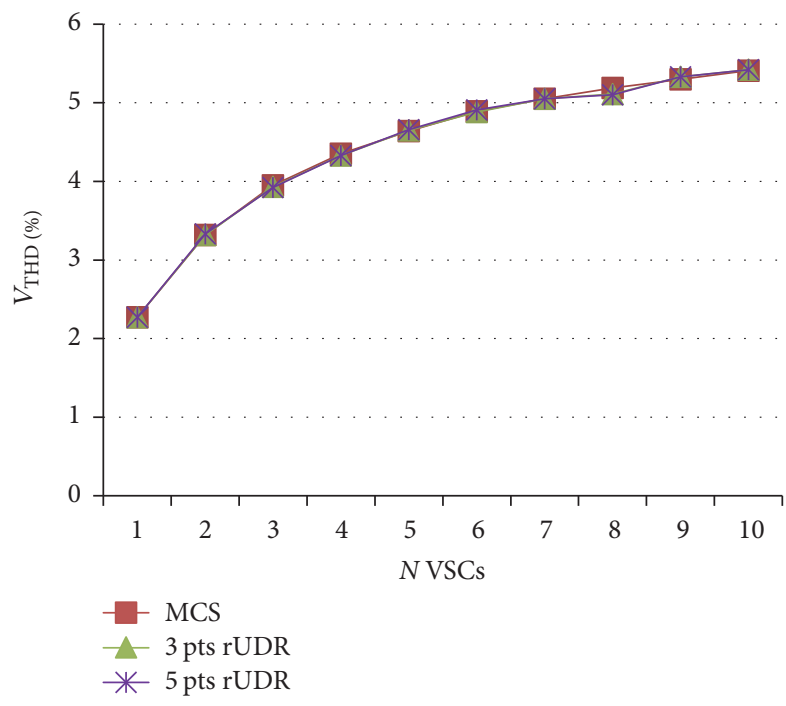

Figure 8: Predicted VgaTHD using MCS, rUDR 3 pts, and rUDR 5 pts under Power Variation.

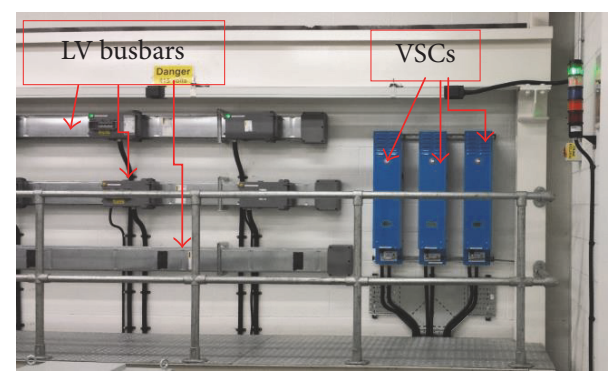

FIGURE 9: Laboratory setup showing 415LV busbars and 3 VSCs.

power/voltage supply (Figure 10) as the RES source, current and voltage measuring device, and 3 isolating transformers.

The 3 VSCs are connected in parallel and supplied from the mains through a 3-phase distribution line and a transformer. The VSCs are isolated using 3 transformers at the grid side of the VSC and connected to the grid side of the programmable power/voltage supply (RES source).

The isolating transformers are necessary to protect the equipment and also to prevent short circuit. The main utility feed represents the grid so the VSCs are operating in the grid tied mode. The voltage and current measurements were done with LabView on a CompactRIO using NI-9227 and NI-9225 units; the current is passed through 100:5 CTs prior to feeding it into the measuring blocks. The time taken to conduct each practical session is presented in Table 2, where E-MCS refers to the Experimental MCS styled approach.

The internal components of the VSCs in Figure 9 were not simulated because of Intellectual Property (IP) protection.

7.1. Case Study 1: Prediction under Uniform Power Variation. Output Power Variations have been earlier shown to affect THD and this study aims to predict the net harmonics of 3 VSCs in a case where the output power uniformly varies randomly and independently (range: $2.5 \mathrm{~kW}-10.0 \mathrm{~kW}$ ).
TABLE 1: Microgrid and VSC laboratory parameters.

\begin{tabular}{lc}
\hline VSC Parameter & \\
Rated power, $P$ & $12.5 \mathrm{~kW}$ \\
Rated line voltage & $415 \mathrm{~V}$ \\
Rated current & $32 \mathrm{~A}$ \\
VSC topology & 3-phase 2-level VSC \\
VSC filter configuration & $\mathrm{LCL}$ \\
\hline Electrical power system & \\
Mains line voltage & $415 \mathrm{~V}$ \\
Current & Fuse $20 \mathrm{~A}$ \\
\hline RES source (programmable & \\
power/voltage supply) & \\
Rated power & $90 \mathrm{~kW}$ \\
Rated voltage & $1000 \mathrm{~V}$ \\
Rated current & $300 \mathrm{~A}$ \\
Fuse rating & $125 \mathrm{~A}$ \\
\hline
\end{tabular}

Statistical power variation

Uniform power variation

Range $=2.5 \mathrm{~kW}-10.0 \mathrm{~kW}$

Normal power variation

Mean $=6.25 \mathrm{~kW}$

Mean $=3.44 \mathrm{~kW}$

$\operatorname{Std}=2.02$

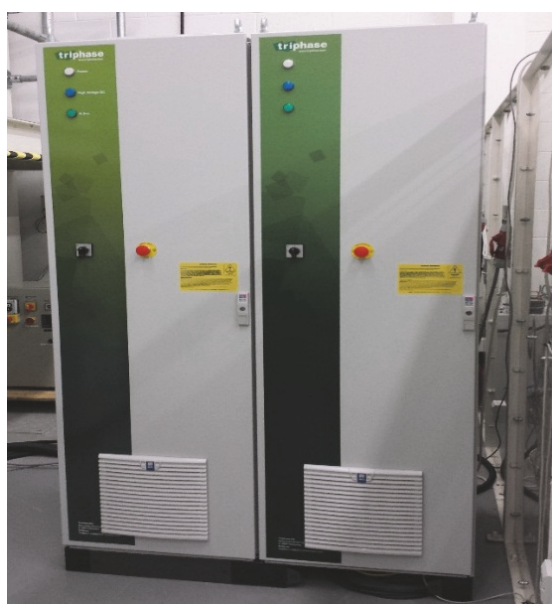

Figure 10: Controllable power and voltage source (Triphase).

The VSCs were first varied with thousands of random power input values to imitate the MCS approach. Then the net harmonics are measured and recorded. The UDR sigma points and weights also generated. These were fed into the VSCs as power inputs and the net harmonics measured at the PCC. The results of the E-MCS styled approach and the UDR are then statistically analyzed and compared to measure the efficiency of the UDR technique. The results are given in Table 3 and Figures 11 and 12.

It can be seen in Table 3 and Figures 11 and 12 that the UDR predicted results for $I_{\mathrm{THD}}$ and $V_{\mathrm{THD}}$ have a good match with the E-MCS approach. 
TABLE 2: Approximate run time for each practical session.

\begin{tabular}{lcccc}
\hline & \multicolumn{2}{c}{ Case 1 } & \multicolumn{2}{c}{ Case 2 } \\
& E-MCS & UDR 5 pts & E-MCS & UDR 5 pts \\
\hline Time (s) & 21600 & 2700 & 24200 & 2850 \\
Time saved (\%) & - & 88 & - & 88 \\
\hline
\end{tabular}

TABLE 3: THD result of VSCs under uniform variation of power.

\begin{tabular}{lcccc}
\hline & \multicolumn{2}{c}{$I_{\text {THD }}(\%)$} & \multicolumn{2}{c}{$V_{\text {THD }}(\%)$} \\
& Mean & Std & Mean & Std \\
\hline E-MCS & 4.46 & 1.44 & 1.93 & 0.52 \\
UDR & 5.07 & 1.67 & 2.06 & 0.60 \\
Diff & 0.61 & 0.23 & 0.13 & 0.08 \\
\hline
\end{tabular}

TABLE 4: THD result of VSCs under normal variation of power.

\begin{tabular}{lcccc}
\hline & \multicolumn{2}{c}{$I_{\text {THD }}(\%)$} & \multicolumn{2}{c}{$V_{\text {THD }}(\%)$} \\
& Mean & Std & Mean & Std \\
\hline E-MCS & 4.61 & 1.62 & 1.95 & 0.51 \\
UDR & 4.72 & 1.19 & 2.25 & 0.54 \\
Diff & 0.11 & -0.43 & 0.30 & 0.03 \\
\hline
\end{tabular}

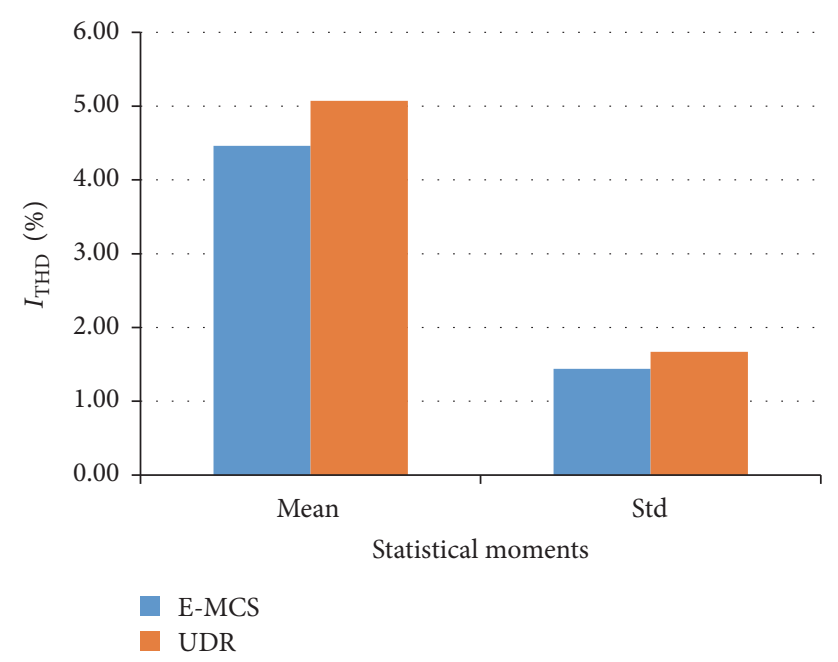

FIgure 11: Predicted IgaTHD using E-MCS and rUDR 5 pts for Uniform Power Variation.

7.2. Case Study 2: Prediction under Normal Power Variation. In this case study, the output power was varied randomly following a Gaussian distribution (mean value $=3.44 \mathrm{~kW}$ and Std $=2.02 e+3$ ). Just as in Case 1 , the VSCs power was varied with thousands of random power inputs to mimic the MCS approach. The statistical information of the distribution is recorded and the THD measured. Then the 5 pts UDR sigma points and weights were generated and inputted to evaluate the performance of the UDR technique using the E-MCS as a benchmark. The results are given in Table 4 and Figures 13 and 14.

Table 4 and Figures 13 and 14 show a good match between the UDR and the E-MCS mean values for $I_{\mathrm{THD}}$ and $V_{\mathrm{THD}}$.

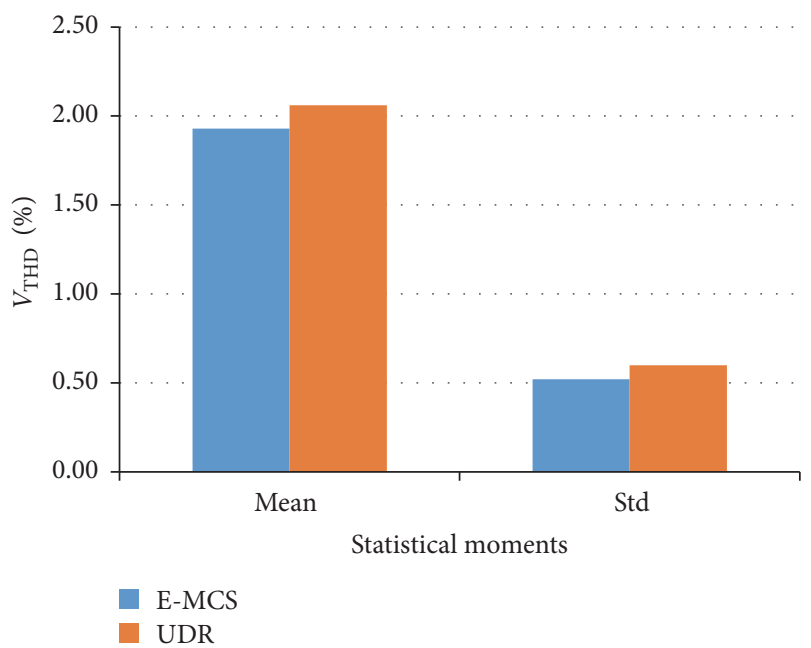

FIgure 12: Predicted VgaTHD using E-MCS and rUDR 5 pts for Uniform Power Variation.

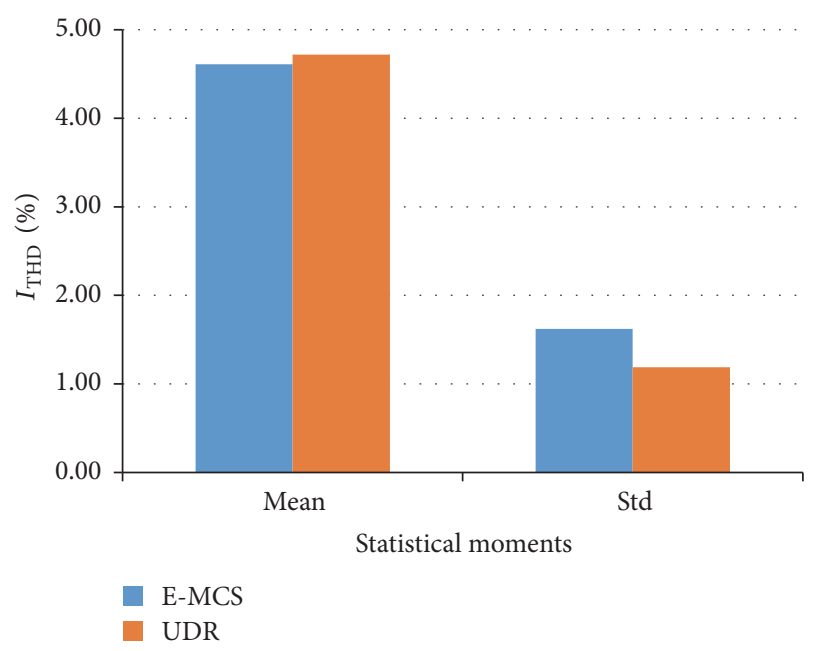

Figure 13: Predicted IgaTHD using E-MCS and rUDR 5 pts for Gaussian Power Variation.

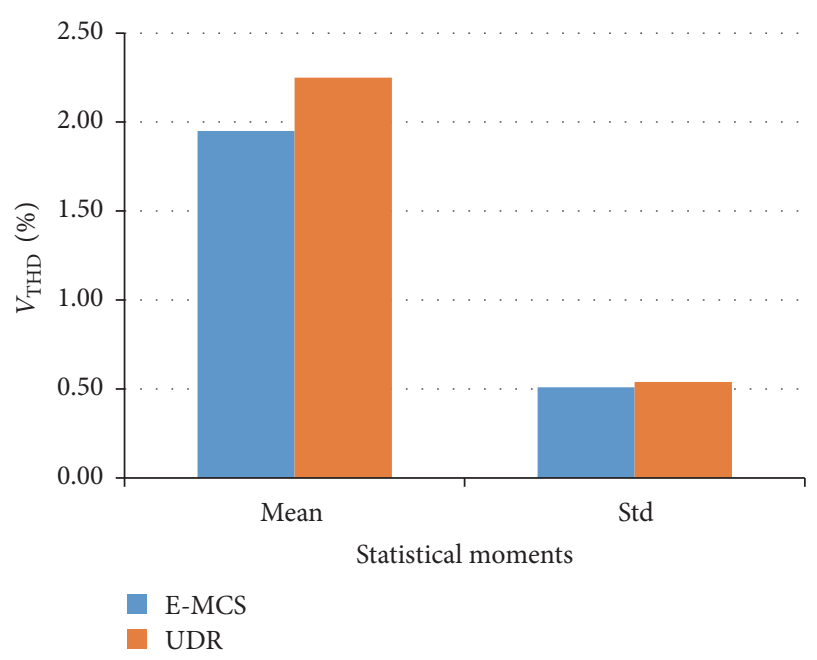

Figure 14: Predicted VgaTHD using E-MCS and rUDR 5 pts for Gaussian Power Variation. 


\section{Conclusion}

Current and voltage harmonic distortion level of many connected converters have been successfully predicted using the 3 and 5 points' approximated Univariate Dimension Reduction (UDR) technique. The effects of the variations in filter inductor value and operating power on harmonic distortion levels of VSCs at the PCC of an EPS were also presented. The EPS test system was designed using PLECS and MATLAB simulation tools.

The proposed prediction method (UDR) ensured full interactions between the harmonic sources (VSCs) and the entire EPS in predicting the THD at the PCC unlike most analytical techniques where the harmonic sources are assumed to be independent.

It was observed from the results that the 3 and 5 points' reduced UDR (rUDR) technique effectively predicted the mean and standard deviation of the harmonics at the PCC of the EPS and can be used as an alternative predictive tool for the Monte Carlo Simulation approach. One significant advantage of the rUDR highlighted in this study is its drastic reduction of computational time and burden in predicting harmonics. The UDR technique was also experimentally validated and the results obtained were in good agreement with the E-MCS styled approach.

The study showed that the reduced UDR can be used by power companies/design engineers in the choice of parameters since the effects of real world uncertainties possible in the operation of modern EPS are being taken into account. It also provides possible outcomes of variation of design parameter/system characteristics on the generated harmonics of an EPS containing multiple power converters. This technique can be further utilized on other power converters, nonlinear loads, and harmonic sources and can also be applied to predict other harmonic indices like the individual harmonic distortions of currents and voltages.

\section{Conflicts of Interest}

The authors declare that there are no conflicts of interest regarding the publication of this paper.

\section{Acknowledgments}

This manuscript is derived from the following thesis. P. M. Ivry, "Predicting Stochastic Harmonics of Multiple Converters in a Power System (Microgrid)," Department of Electrical and Electronic Engineering, University of Nottingham, Nottingham, 2016. The first author would like to acknowledge the Petroleum Technology Development Fund (PTDF), Nigeria, for supporting this research.

\section{References}

[1] A. Yazdani and R. Iravani, Voltage-Sourced Converters in Power Systems: Modeling, Control, and Applications, John Wiley and Sons, 2010.

[2] N. Mohan, T. M. Undeland, and W. P. Robbins, Power Electronics, Converters, Applications and Design, John Wiley and Sons, Third edition, 2003.
[3] P. M. Ivry, M. J. Rawa, D. W. P. Thomas, and M. Sumner, "Power quality of a voltage source converter in a smart grid," in Proceedings of the IEEE Grenoble Conference PowerTech (POWERTECH '13), Grenoble, France, 2013.

[4] Y. Baghzouz, R. F. Burch, A. Capasso et al., "Time-varying harmonics: part I: characterizing measured data," IEEE Transactions on Power Delivery, vol. 13, no. 3, pp. 938-944, 1998.

[5] M. H. Kalos and P. A. Whitlock, Monte Carlo Methods, WileyVCH Verlag GmbH \& Co. KGaA, Weinheim, Germany, 2nd edition, 2008.

[6] J. He, J. Jiang, J. Huang, and W. Chen, "Model of EMI coupling paths for an off-line power converter", in Proceedings of the 19th Annual IEEE Applied Power Electronics Conference and Exposition (APEC '04), vol. 2, pp. 708-713, Anaheim, Calif, USA, 2004.

[7] M. Ferber, C. Vollaire, L. Krähenbühl, J.-L. Coulomb, and J. A. Vasconcelos, "Conducted EMI of DC-DC converters with parametric uncertainties," IEEE Transactions on Electromagnetic Compatibility, vol. 55, no. 4, pp. 699-706, 2013.

[8] S. R. Kaprielian, A. E. Emanuel, R. V. Dwyer, and H. Mehta, "Predicting voltage distortion in a system with multiple random harmonic sources," IEEE Transactions on Power Delivery, vol. 9, no. 3, pp. 1632-1638, 1994.

[9] P. T. Staats, W. M. Grady, A. Arapostathis, and R. S. Thallam, "A statistical method for predicting the net harmonic currents generated by a concentration of electric vehicle battery chargers," IEEE Transactions on Power Delivery, vol. 12, no. 3, pp. 1258$1264,1997$.

[10] K.-H. Liu, "Analysis of probabilistic harmonic currents and voltages of electronic power converter contributed in distribution system," Research Journal of Applied Sciences, Engineering and Technology, vol. 5, no. 4, pp. 1263-1270, 2013.

[11] J.-H. Teng, R.-C. Leou, C.-Y. Chang, and S.-Y. Chan, "Harmonic current predictors for wind turbines," Energies, vol. 6, no. 3, pp. 1314-1328, 2013.

[12] Y. G. Hegazy and M. M. A. Salama, "Calculations of diversified harmonic currents in multiple converter systems," in Proceedings of the Power Engineering Society Summer Meeting, vol. 2, pp. 727-731, Seattle, Washington DC, USA, 2000.

[13] Y. J. Wang and L. Pierrat, "Summation of harmonic currents produced by AC/DC static power converters with randomly fluctuating loads," IEEE Transactions on Power Delivery, vol. 9, no. 2, pp. 1129-1135, 1994.

[14] E. Ngandui, E. J. Mohammed, and A. Cheriti, "Prediction of harmonics produced by multiple variable speed drives with randomly fluctuating loads," in Proceedings of the CCECE 2000Canadian Conference on Electrical and Computer Egineering, vol. 2, pp. 1157-1161, Seattle, Washington DC, USA, 2000.

[15] H. V. Haghi and M. T. Bina, "A study on probabilistic evaluation of harmonic levels produced by static compensators," in Proceedings of the Australasian Universities Power Engineering Conference, pp. 1-6, Sydney, Australia, 2008.

[16] D. G. Infield, P. Onions, A. D. Simmons, and G. A. Smith, "Power quality from multiple grid-connected single-phase inverters," IEEE Transactions on Power Delivery, vol. 19, no. 4, pp. 1983-1989, 2004.

[17] MathWorks, "Monte Carlo simulation," 09 March, 2015, http:// uk.mathworks.com/discovery/monte-carlo-simulation.html.

[18] P. Jorgensen, J. Christensen, and J. Tande, "Probabilistic load flow calculation using Monte Carlo techniques for distribution network with wind turbines," in Proceedings of the International 
Conference on Harmonics and Quality of Power, vol. 2, pp. 11461151, Athens, Greece, 1998.

[19] D. W. P. Thomas, O. A. Oke, L. R. A. X. De Menezes, and C. Christopoulos, "The use of unscented transforms in modeling the statistical response of nonlinear scatterer in a reverberation chamber," in Proceedings of the 30th URSI General Assembly and Scientific Symposium (URSIGASS '11), pp. 1-4, Turkey, 2011.

[20] L. R. A. X. De Menezes, D. W. P. Thomas, C. Christopoulos, A. Ajayi, and P. Sewell, "The use of unscented transforms for statistical analysis in EMC," in Proceedings of the IEEE International Symposium on Electromagnetic Compatibility (EMC '08), pp. 15, Hamburg, Germany, 2008.

[21] J. M. Morales and J. Pérez-Ruiz, "Point estimate schemes to solve the probabilistic power flow," IEEE Transactions on Power Systems, vol. 22, no. 4, pp. 1594-1601, 2007.

[22] O. A. Oke, Enhanced Unscented Transform Method for Probabilistic Load Flow Studies, Electrical and Electronics Engineering, University of Nottingham, Nottingham, UK, 2013.

[23] S. Rahman and $\mathrm{H}$. Xu, "A univariate dimension-reduction method for multi-dimensional integration in stochastic mechanics," Probabilistic Engineering Mechanics, vol. 19, no. 4, pp. 393-408, 2004.

[24] K. H. Ahmed, S. J. Finney, and B. W. Williams, "Passive filter design for three-phase inverter interfacing in distributed generation," in Proceedings of the 5th International ConferenceWorkshop Compatibility in Power Electronics (CPE '07), pp. 1-9, Poland, 2007.

[25] M. Liserre, F. Blaabjerg, and A. Dell'aquila, "Step-by-step design procedure for a grid-connected three-phase PWM voltage source converter," International Journal of Electronics, vol. 91, no. 8, pp. 445-460, 2004.

[26] A. A. Rockhill, M. Liserre, R. Teodorescu, and P. Rodriguez, "Grid-filter design for a multimegawatt medium-voltage voltagesource inverter," IEEE Transactions on Industrial Electronics, vol. 58, no. 4, pp. 1205-1217, 2011.

[27] R. Teodorescu, M. Liserre, and P. Rodríguez, Grid Converters for Photovoltaic and Wind Power Systems, John Wiley and Sons, 2011.

[28] I. Sefa, N. Altin, and S. Ozdemir, "An implementation of grid interactive inverter with reactive power support capability for renewable energy sources," in Proceedings of the 3rd IEEE International Conference on Power Engineering, Energy and Electrical Drives (PowerEng '11), pp. 1-6, Spain, 2011.

[29] P. M. Ivry, Predicting stochastic harmonics of multiple converters in a power system (microgrid) [Ph.D. thesis], Electrical and Electronic Engineering, University of Nottingham, Nottingham, UK, 2016.

[30] D. W. P. Thomas, O. A. Oke, and C. Smartt, "Statistical analysis in EMC using dimension reduction methods," in Proceedings of the IEEE International Symposium on Electromagnetic Compatibility (EMC '14), pp. 316-321, Raleigh, NC, USA, 2014.

[31] O. A. Oke, D. W. P. Thomas, and G. M. Asher, "A new probabilistic load flow method for systems with wind penetration," in Proceedings of the IEEE PES Trondheim PowerTech: The Power of Technology for a Sustainable Society (POWERTECH '11), pp. 1-6, Norway, 2011.

[32] W. Gautschi, Numerical Mathematics and Scientific Computation, Oxford University Press, Oxford, UK, 2004.

[33] H. Xu and S. Rahman, "A generalized dimension-reduction method for multidimensional integration in stochastic mechanics," International Journal for Numerical Methods in Engineering, vol. 61, no. 12, pp. 1992-2019, 2004.
[34] I. Lee, K. K. Choi, L. Du, and D. Gorsich, "Dimension reduction method for reliability-based robust design optimization," Computers and Structures, vol. 86, no. 13-14, pp. 1550-1562, 2008.

[35] B. Zou and Q. Xiao, "Probabilistic load flow computation using univariate dimension reduction method," International Transactions on Electrical Energy Systems, vol. 24, no. 12, pp. 1700-1714, 2014.

[36] F. J. Ruiz-Rodriguez, J. C. Hernández, and F. Jurado, "Probabilistic load flow for photovoltaic distributed generation using the Cornish-Fisher expansion," Electric Power Systems Research, vol. 89, pp. 129-138, 2012.

[37] H. Bayem, M. Petit, P. Dessante, F. Dufourd, and R. Belhomme, "Probabilistic characterization of wind farms for grid connection studies," in Proceedings of the European Wind Energy Conference and Exhibition 2007 (EWEC '07), pp. 1482-1489, Tampa, Fla, USA, 2007.

[38] PLECS 3.5.1, “Plexim GmbH," Zurich, 2014.

[39] MATLAB 8.1.0.604 (R2013a), MathWorks, Inc., 2013.

[40] P. M. Ivry, M. J. Rawa, and D. W. P. Thomas, "Factors affecting the harmonics generated by a generic voltage source converter within a microgrid," in Proceedings of the Saudi Arabia Smart Grid Conference, Jeddah, 2012, pp. B-65.

[41] E. E. Ahmed and W. Xu, "Assessment of harmonic distortion level considering the interaction between distributed threephase harmonic sources and power grid," IET Generation, Transmission and Distribution, vol. 1, no. 3, pp. 506-515, 2007.

[42] A. Mansoor, W. M. Grady, A. H. Chowdhury, and M. J. Samotyj, "An investigation of harmonics attenuation and diversity among distributed single-phase power electronic loads," IEEE Transactions on Power Delivery, vol. 10, no. 1, pp. 467-473, 1995. 


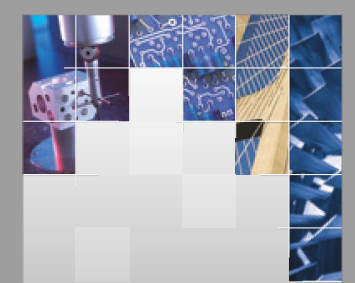

\section{Enfincering}
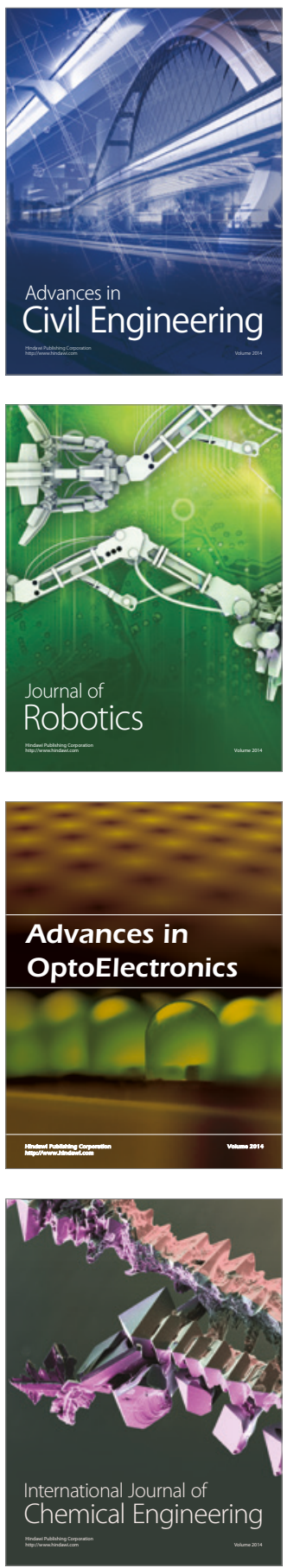

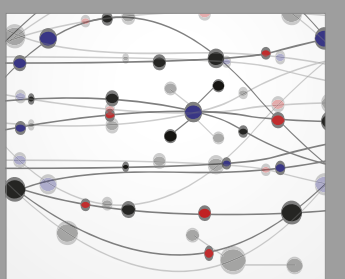

The Scientific World Journal

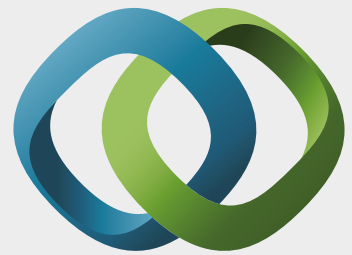

\section{Hindawi}

Submit your manuscripts at

https://www.hindawi.com
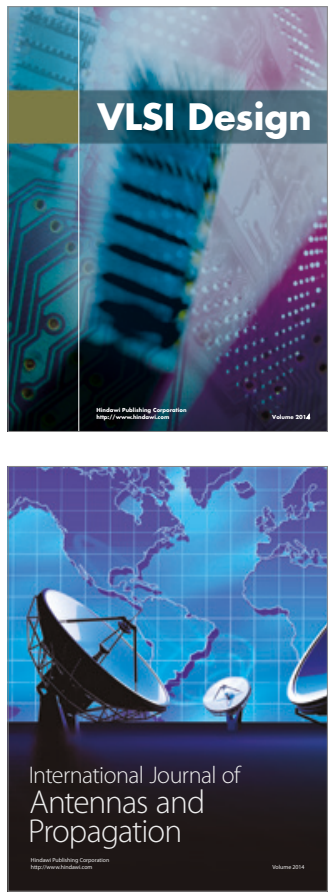

\section{Rotating}

Machinery
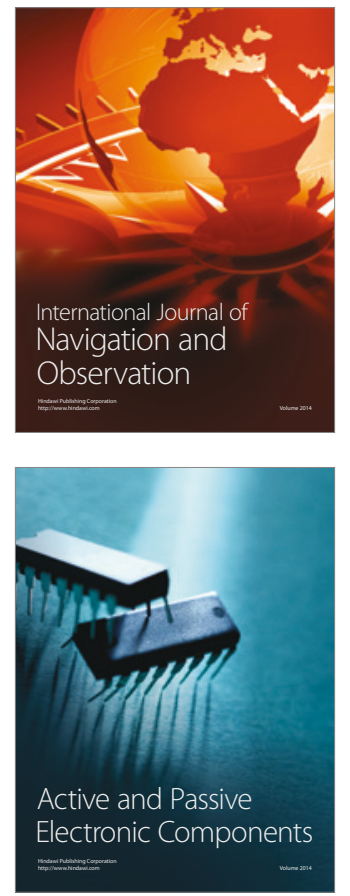
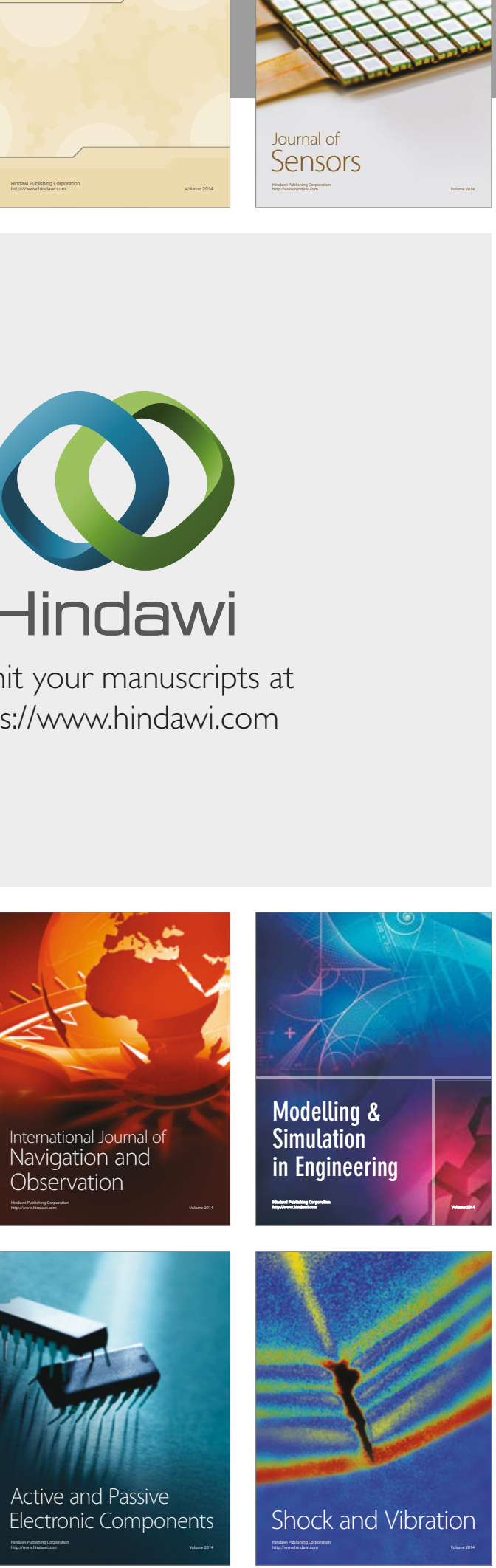
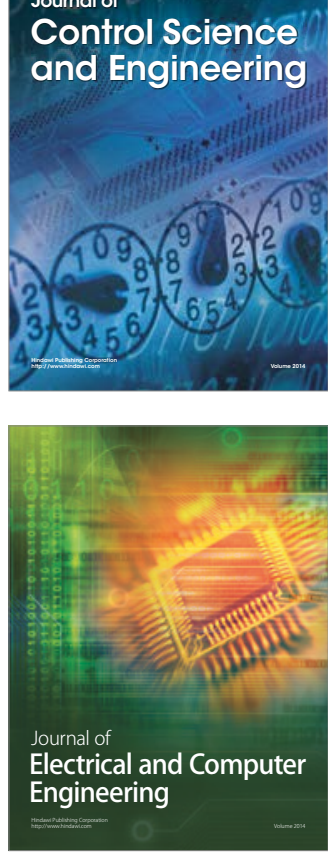

Distributed

Journal of

Control Science

and Engineering
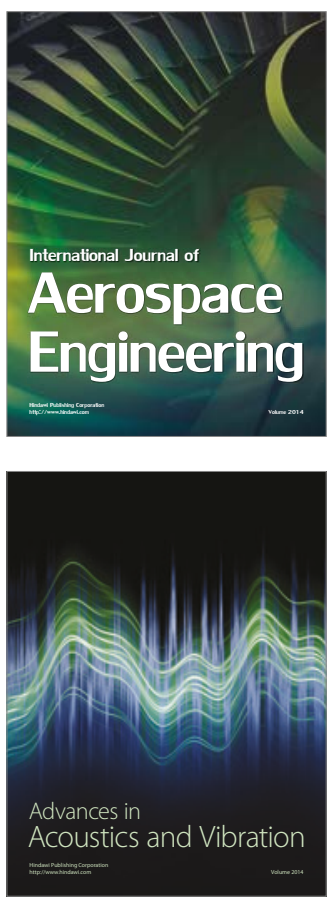

Sensor Networks 